\title{
Case of Unilateral Peripheral Cone Dysfunction
}

\author{
Yujin Mochizuki $^{\mathrm{a}}$ Kei Shinoda ${ }^{\mathrm{b}}$ Celso Soiti Matsumoto ${ }^{\mathrm{b}}$ \\ Gerd Klose $^{c}$ Emiko Watanabe ${ }^{b}$ Keisuke Seki ${ }^{\text {b }}$ \\ Itaru Kimura ${ }^{a}$ Atsushi Mizota ${ }^{b}$ \\ ${ }^{a}$ Department of Ophthalmology, Juntendo University Urayasu Hospital, Chiba, \\ ${ }^{b}$ Department of Ophthalmology, Teikyo University School of Medicine, \\ University Hospital Itabashi, and ${ }^{\mathrm{C}}$ Carl Zeiss Meditec Co., Ltd., Tokyo, Japan
}

\section{Key Words}

Tunnel vision - Electroretinogram · Multifocal ERG - Optical coherence tomography · Pattern visual evoked potentials

\begin{abstract}
Purpose: Peripheral cone dystrophy is a subgroup of cone dystrophy, and only 4 cases have been reported. We present a patient with unilateral peripheral cone dysfunction and report the functional changes determined by electrophysiological tests and ultrastructural changes determined by spectral domain optical coherence tomography (SD-OCT).

Case: A 34-year-old woman complained of blurred vision in both eyes. Our examination showed that her visual acuity was $0.05 \mathrm{OD}$ and $0.2 \mathrm{OS}$. A relative afferent pupillary defect was present in her right eye. The results of slit-lamp examination, ophthalmoscopy, and fluorescein angiography were normal except for pallor of the right optic disc. SD-OCT showed a diffuse thinning of the retina in the posterior pole of the right eye. A severe constriction of the visual fields was found in both eyes but more in the right eye. The photopic full-field electroretinograms (ERGs) were reduced in the right eye but normal in the left eye. The multifocal ERGs were severely reduced throughout the visual field except in the central area of the right eye. The multifocal ERGs from the left eye were normal. The pattern visual evoked responses were within the normal range in both eyes. She had a 5 -year history of sniffing paint thinner.

Results: Although the visual dysfunction was initially suspected to be due to psychological problems from the results of subjective tests, objective tests indicated a peripheral cone dysfunction in the right eye. The pathophysiological mechanism and the relationship with thinner sniffing were not determined.

Conclusions: Our findings indicate that peripheral cone dysfunction can occur unilaterally. Electrophysiology and SD-OCT are valuable tests to perform to determine the pathogenesis of unusual ocular findings objectively.
\end{abstract}




\section{Introduction}

An inherited central cone dysfunction was named occult macular dystrophy (OMD) or Miyake's disease [1-3], and the gene responsible for OMD has been identified [4]. In $\mathrm{OMD}$, only the central cone function is abnormal. There are also cases where only the peripheral cone functions are abnormal while the central cone function is normal. However, these cases are rare and only four cases of peripheral cone dysfunction have been reported. In these cases, the rod function is normal over the entire retina [5, 6]. Kondo et al. [5] first described peripheral cone dystrophy, and they carefully analyzed focal areas of the central retina in 3 patients with peripheral cone dystrophy. They showed that the dysfunction of the cones was predominantly in the periphery and the central area was relatively well preserved. Okuno et al. [6] reported an elderly man with bilateral peripheral cone dystrophy, and the patient's signs and symptoms had not changed for over 50 years. Therefore they concluded that such condition in their case was not a manifestation of an early stage of the more common type of cone dystrophy.

We present a patient with peripheral cone dysfunction but the abnormalities were found in only one eye. In addition to the unilateral dystrophy, the patient had several features that were different from previous cases of peripheral cone dystrophy.

\section{Case Report}

A 34-year-old Japanese woman complained of a gradually decrease of her vision in both eyes. She visited a neighborhood eye clinic on December 2008, and the examination showed that her bestcorrected decimal visual acuity (BCVA) was 0.3 OD and 0.5 OS. A diagnosis was not made, and she was not treated. Three months later she was referred to our clinic. She reported that she had noticed that her right visual field had been constricted for 10 years. She did not complain of photopsia, photophobia, night blindness, or color vision disturbances. She had a history of paint thinner sniffing of more than 5 years. Our examination showed that her BCVA was $0.05 \mathrm{OD}$ and $0.2 \mathrm{OS}$. The results of slit-lamp biomicroscopy, ophthalmoscopy, and fluorescein angiography were normal except for right optic disc pallor (fig. 1). Goldman and Humphrey visual field tests showed a concentric constriction of the visual fields in both eyes but more severe in the right eye (fig. 1). A relative afferent pupillary defect was present in the right eye. Brain magnetic resonance imaging showed no abnormalities.

The amplitudes of the full-field scotopic b-waves of the ERGs were within normal range in both eyes (fig. 2), and that of the mixed rod:cone ERGs were also within normal range. The oscillatory potentials (OPs) and the photopic cone ERGs were unrecordable in the right eye, i.e., less than the noise level ( $5 \mu \mathrm{V}$ for OPs and $20 \mu \mathrm{V}$ for the photopic cone ERGs), and the flicker ERGs were highly attenuated by approximately $95 \%$ of that of the right eye. The responses from the left eye were essentially normal except that the amplitude of the scotopic rod ERGs was at the lower limit of the normal range.

The multifocal ERGs (mfERGs; fig. 2, online suppl. table 1; for all online suppl. material, see www.karger.com/doi/10.1159/000339129) were well preserved in the central retina but severely reduced in the periphery of the right eye. These findings were in good accordance with the results of perimetry. The mfERGs were well preserved in all areas of the left eye. The mfERGs from each concentric ring were summed and analyzed. This showed that only the central responses were preserved in the right eye, and no significant difference of the implicit time was found in the central area between the two eyes. Pattern visual evoked response was within normal limits in both eyes (fig. 2).

Microstructural images of the fovea were obtained by the SD-OCT (OCT4000, Cirrus HD-OCT, Carl Zeiss Medic Inc., Dublin, Calif., USA). The 3D datasets were obtained using the raster scan protocol of 128 horizontal B-scan images, each composed of 512 axial scans. This raster scan protocol covered an 
area of $6 \mathrm{~mm}$ (horizontal) $\times 6 \mathrm{~mm}$ (vertical) $\times 1.7 \mathrm{~mm}$ (axial) with a horizontal pixel spacing of $11 \mu \mathrm{m}$ $(6 \mathrm{~mm} / 512)$ and a vertical pixel spacing of $47 \mu \mathrm{m}(6 \mathrm{~mm} / 128)$.

High-quality images of $9 \mathrm{~mm}$ horizontal and $6 \mathrm{~mm}$ vertical scans were obtained using the HD 5line raster scan protocol of 5 B-scan images, each composed of 1,024 axial scans. Each B-scan was aquired with 4-times oversampling and subsequent pixel profiling to obtain noise-reduced images. This raster scan protocol examines the cross section of B-scans through the fovea with a horizontal pixel spacing of $9 \mu \mathrm{m}(9 \mathrm{~mm} / 1,024)$ and a vertical pixel spacing of $6 \mu \mathrm{m}(6 \mathrm{~mm} / 1,024)$.

Based on the HD 5-line raster scans in the horizontal and vertical orientation of both the right and left eye, the outer nuclear layer (ONL) could be identified and its thickness determined. The thickness of the ONL was defined as the vertical distance between the highly reflective line corresponding to the external limiting membrane (ELM) and the midpoint of the transition from the highly reflective outer plexiform layer (OPL) to the less reflective ONL. Manual thickness measurements were made at many points on the central horizontal and vertical B-scan cross sections (online suppl. fig. 1) using the builtin caliper tool. In addition, the images were analyzed with the help of an external mathematical program to automatically detect the ONL boundaries and determine the layer thickness. The thickness values have been averaged over segments of $1 \mathrm{~mm}$ length, corresponding to the popular EDTRS grid.

In general, the configuration and microstructure in the macula were normal in both eyes; the ELM, the photoreceptor inner and outer segment (IS/OS) lines, and the foveal bulge were distinct in the SDOCT images in both eyes (fig. 1). However, comparisons of each layer between the two eyes showed a diffuse thinning of the retina especially the ONL in the extramacular area of the right eye. Online supplementary table 2 summarizes the quantitative results obtained from the manual and automated thickness analysis.

\section{Discussion}

Peripheral cone dystrophy is a very rare clinical entity, and its clinical characteristics have been presented in only two publications $[5,6]$. The possibility of having patients with peripheral cone dystrophy or patients with similar characteristics has been discussed [7-10]. However, these authors did not compare the central and peripheral cone function and also did not demonstrate that the rod system was functioning normally across the retina.

Recent developments of the clinical techniques of evaluating retinal function in localized areas, e.g., focal ERGs, mfERGs, and rod and cone perimetry, have allowed assessments of central [1-3] or peripheral cone dysfunction [5, 6]. The etiology of the findings of our case is unknown. Inherited diseases, vascular disease, systemic disease, infection, inflammation, and an exposure to toxins were considered in the differential diagnosis. Our case had a history of paint thinner sniffing, and thus the results of subjective testing should be carefully interpreted. However, the electrophysiological findings including full-field ERGs, mfERGs, and visual evoked potentials clearly showed diffuse cone dysfunction except in the central area of only the right eye. On the other hand,rod in the right eye, both rod and cone in the left eye, and optic nerve functioned well in both eyes. Moreover microstructural analysis on the retina supports these objective functional results. However, the severely decreased visual acuity and highly constricted visual field in both eyes do not agree with our diagnosis of peripheral cone dystrophy. These contradictory findings might be because our patient had psychogenic disorders induced by thinner sniffing and the possibility of unilaterally pronounced toxic effects cannot be denied [11]. But thinner sniffing has been reported to lead to mainly optic neuropathy $[12,13]$ but the normal visual evoked potentials of our patient indicated that this was not likely. 
Another differential diagnosis of our case is acute zonal occult outer retinopathy (AZOOR) which is a uni- or bilateral disease characterized by an acute zonal loss of outer retinal function with minimal ophthalmoscopic changes [14]. Recently, local retinal microstructure assessed by Fourier-domain and spectral-domain optical coherence tomography showed that the major OCT findings in this disease were the selective absence or discontinuity of the IS/OS line and the cone outer segment tip (COST) line in the retinal areas corresponding to the functional decrease $[15,16]$. Defect of the IS/OS line and thinning of the ONL has been reported in AZOOR $[17,18]$. In our case, the onset was not acute according to the patient's description, but otherwise the clinical manifestations are compatible to AZOOR clinical entity. However, we believe that our case was not AZOOR because the IS/OS line appeared normal in the area with severely reduced mfERGs in the right eye, and a unilateral, diffuse, peripheral, cone-selective dysfunction in AZOOR has not been reported.

Microstructural analyses showed a selective thinning of the ONL with the outer plexiform layer and IS/OS line intact. These findings are in good agreement with the severe attenuation of the a-wave in photopic ERG and mfERG. This supports the idea of a selective cone dysfunction.

Only five cases of unilateral cone dysfunction have been reported [19-22]. Two cases from Sieving [19] had normal rod ERGs in both eyes but reduced cone ERGs in only one eye. He reported abnormally prolonged $b$-wave when long-duration photopic flashes were used, and these abnormalities were ascribed to the hyperpolarizing bipolar and/or horizontal cells. In contrast, our subject had no measurable full-field photopic ERGs. The other three cases, a 19-year-old woman reported by Zervas and Smith [20], a 53-year-old woman by Brigell and Celesia [21], and a 20-year-old woman by Nomura et al. [22], had unilateral severe cone dysfunction while the rod function was intact in both eyes, which is similar with our case. But all three cases had an acute onset and the 20-year-old woman demonstrated bull's eye maculopathy three months later. The two cases from Zervas and Smith, and Brigell and Celesia had good visual acuity and ophthalmoscopically normal macula in the affected eye. Although information about focal macular ERGs was not reported in these cases, the abnormal full-field ERGs and preserved macular function appear similar to our case, except that our case had severely reduced visual acuity.

Considering all of our findings, we conclude that our case had unilateral peripheral cone dysfunction, and the findings are different from those of the more common cone dystrophy as well as of the reported cases with peripheral cone dystrophy.

\section{Disclosure Statement}

No author has a financial or proprietary interest in any material or method mentioned except that G.K. is employed at Carl Zeiss Meditec Co., Ltd., Japan (support limited to not-commercially available data analysis). Support of this study was provided by Research Grants on Sensory and Communicative Disorders from the Ministry of Health, Labor, and Welfare, Japan. 


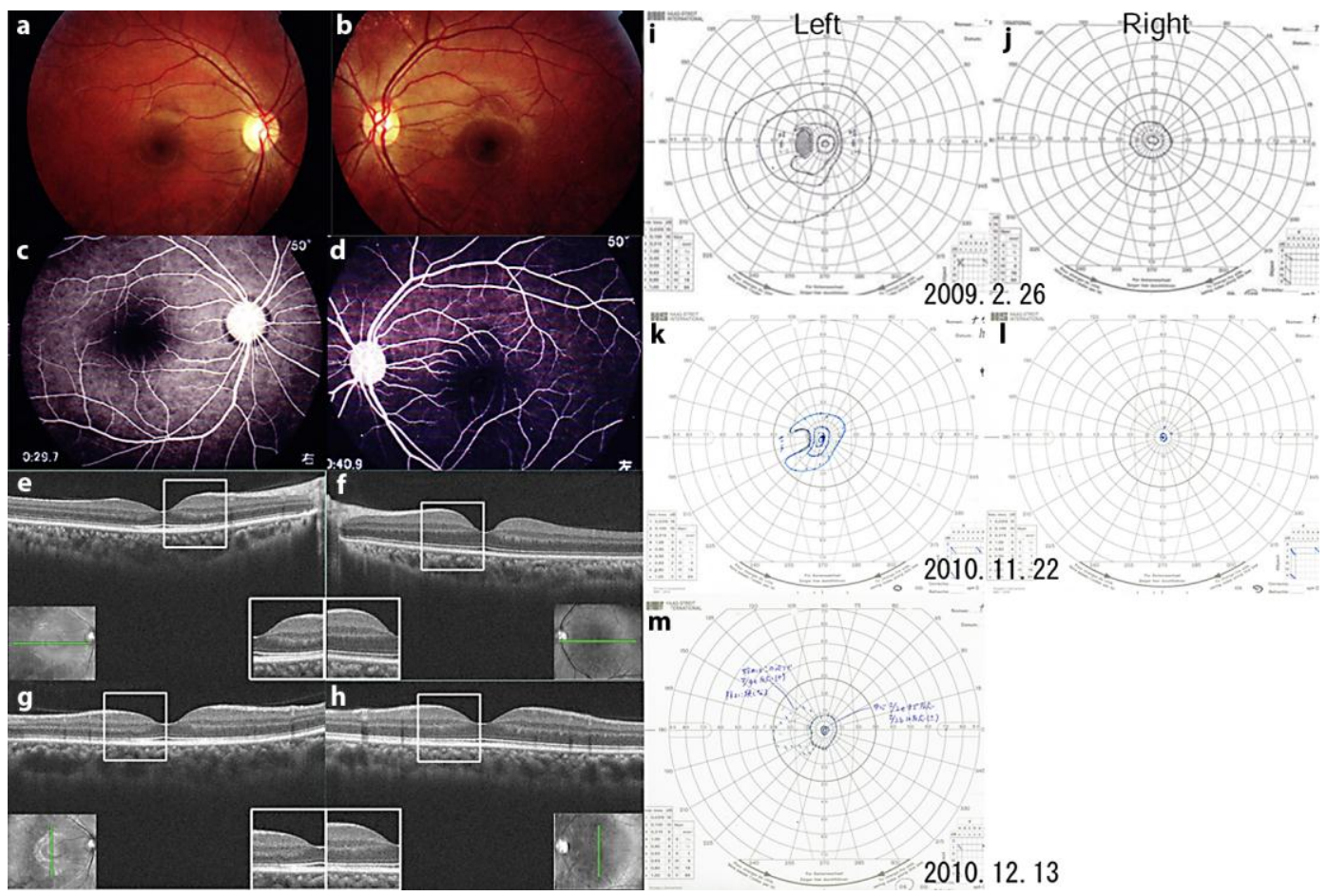

Fig. 1. Fundus photographs, fluorescein angiograms, visual fields, and optical coherence tomographic (OCT) images from our patient with unilateral peripheral cone dystrophy. a-d Fundus photographs and fluorescein angiograms showing no abnormal findings. e-h OCT images from a 9-mm horizontal scan of the right (e) and left (f) eyes and vertical scan in the right (g) and left (h) eyes. Note that the outer nuclear layer is thinner in the right eye than that in the left eye in large areas of the retinal except the fovea (inset). i-m Goldmann visual fields showing bilateral constriction of the visual fields in both eyes but more severe in the right eye. 
rod

\section{mixed} rod \& cone

ops

cone

$30 \mathrm{~Hz}$

flicker

VEP
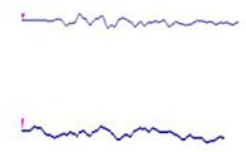

Right
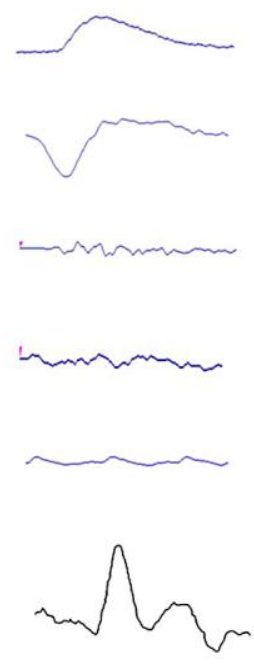

Left
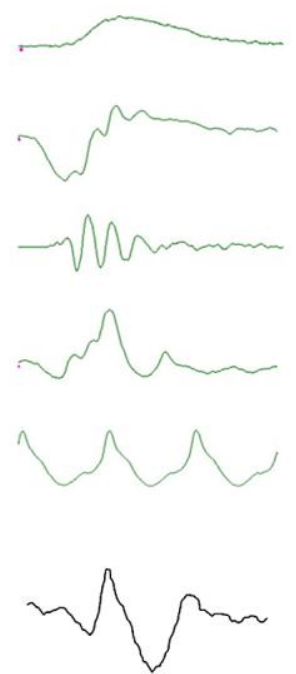

b Left
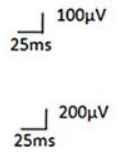

$\perp^{25 \mu \mathrm{V}}$

$25 \mathrm{~ms}$

${\underset{50 \mathrm{~ms}}{100 \mu \mathrm{V}}}^{100}$

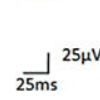

Right
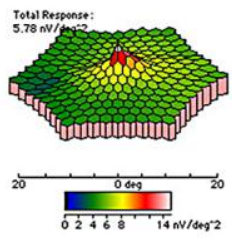

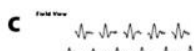

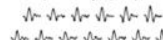
and a d a d s d d d d

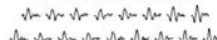

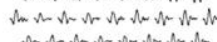

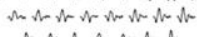

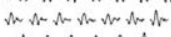

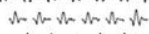
$2 \underset{\mathrm{ms}}{25 \mu \mathrm{V}}$ :men an -1
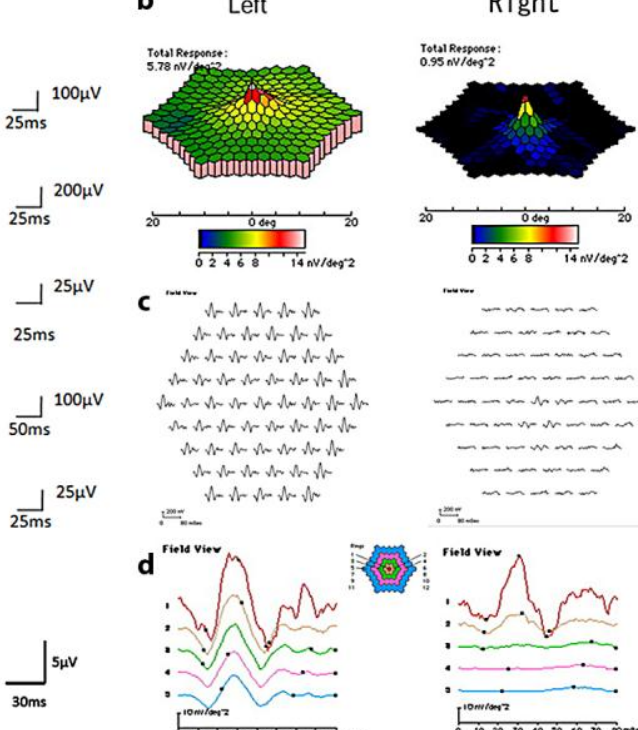

……
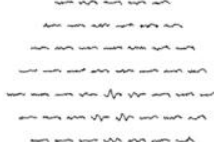

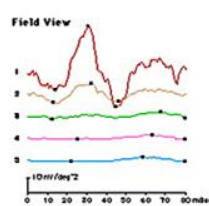

Fig. 2. Full-field and multifocal electroretinograms (ERGs) recorded from our patient with unilateral peripheral cone dystrophy. Full-field ERG showed that rod responses are symmetrical in both eyes, but the responses of cone system are flat in the right eye while normal in the left eye. The multifocal ERGs are severely attenuated in the peripheral retinal area while that from the central area is normal in the right eye. The response density in the left eye was normal.

\section{References}

1 Miyake Y, Ichikawa K, Shiose Y, Kawase Y: Hereditary macular dystrophy without visible fundus abnormality. Am J Ophthalmol 1989;108:292-299.

-2 Miyake Y, Horiguchi M, Tomita N, Kondo M, Tanikawa A, Takahashi H, Suzuki S, Terasaki H: Occult macular dystrophy. Am J Ophthalmol 1996;122:644-653.

3 Miyake Y: Electrodiagnosis of Retinal Disease. Tokyo, Springer-Verlag, 2006.

-4 Akahori M, Tsunoda K, Miyake Y, Fukuda Y, Ishiura H, Tsuji S, Usui T, Hatase T, Nakamura M, Ohde H, Itabashi T, Okamoto $\mathrm{H}$, Takada Y, Iwata T: Dominant mutations in RP1L1 are responsible for occult macular dystrophy. Am J Hum Genet 2010;87:424-429.

5 Kondo M, Miyake Y, Kondo N, Ueno S, Takakuwa H, Terasaki H: Peripheral cone dystrophy: a variant of cone dystrophy with predominant dysfunction in the peripheral cone system. Ophthalmology 2004;111:732-739.

6 Okuno T, Oku H, Kurimoto T, Oono S, Ikeda T: Peripheral cone dystrophy in an elderly man. Clin Experiment Ophthalmol 2008;36:897-899.

$>7$ Krill AE, Deutman AF, Fishman M: The cone degenerations. Doc Ophthalmol 1973;35:1-80.

-8 Pinckers A, Deutman AF: Peripheral cone disease. Ophthalmologica 1977;174:145-150.

$>9$ Pearlman JT, Owen WG, Brounley DW, Sheppard JJ: Cone dystrophy with dominant inheritance. Am J Ophthalmol 1974;77:293-303.

10 Noble KG, Siegel IM, Carr RE: Progressive peripheral cone dysfunction. Am J Ophthalmol 1988;106:557560.

11 Toyonaga N, Adachi-Usami E, Yamazaki H: Clinical and electrophysiological findings in three patients with toluene dependency. Doc Ophthalmol 1989;73:201-207.

-12 Kohriyama K, Hori H, Murai Y, Ninomiya H, Tsukamoto Y: Optic neuropathy induced by thinner sniffing. J UOEH 1989;11:449-453. 
13 Poblano A, Lope Huerta M, Martínez JM, Falcón HD: Pattern-visual evoked potentials in thinner abusers. Arch Med Res 1996;27:531-533.

14 Gass JD: Acute zonal occult outer retinopathy. Donders Lecture: The Netherlands Ophthalmological Society, Maastricht, Holland, June 19, 1992. J Clin Neuroophthalmol 1993;13:79-97.

15 Park SJ, Woo SJ, Park KH, Hwang JM, Chung H: Morphologic photoreceptor abnormality in occult macular dystrophy on spectral-domain optical coherence tomography. Invest Ophthalmol Vis Sci 2010;51:36733679.

16 Sugahara M, Shinoda K, Matsumoto CS, Satofuka S, Hanazono G, Imamura Y, Mizota A: Outer retinal microstructure in a case of acute idiopathic blind spot enlargement syndrome. Case Report Ophthalmol 2011;2:116-122.

17 Spaide RF, Koizumi H, Freund KB: Photoreceptor outer segment abnormalities as a cause of blind spot enlargement in acute zonal occult outer retinopathy-complex diseases. Am J Ophthalmol 2008;146:111120.

18 Ohta K, Sato A, Fukui E: Spectral domain optical coherence tomographic findings at convalescent stage of acute zonal occult outer retinopathy. Clin Ophthalmol 2009;3:423-428.

19 Sieving PA: Unilateral cone dystrophy: ERG changes implicate abnormal signaling by hyperpolarizing bipolar and/or horizontal cells. Trans Am Ophthalmol Soc 1994;92:459 -471; discussion 471-474.

20 Zervas JP, Smith JL: Neuro-ophthalmic presentation of cone dysfunction syndromes in the adult. J Clin Neuroophthalmol 1987;7:202-218.

21 Brigell M, Celesia GG: Electrophysiological evaluation of the neuro-ophthalmology patient: an algorithm for clinical use [review]. Semin Ophthalmol 1992;7:65-78.

22 Nomura R, Kondo M, Tanikawa A, Yamamoto N, Terasaki H, Miyake Y: Unilateral cone dysfunction with bull's eye maculopathy. Ophthalmology 2001;108:49-53. 